\title{
Mathematical Modeling for Sustainable Production Line
}

\author{
Zahra Ghorbani Ravand ${ }^{1}$, \& Qi Xu ${ }^{1}$ \\ ${ }^{1}$ Department of logistics and electronic commerce, Glorious Sun School of Business and Management, Shanghai \\ Donghua University, China \\ Correspondence: Zahra Ghorbani Ravand, Department of logistics and electronic commerce, Glorious Sun \\ School of Business and Management, Shanghai Donghua University, China.
}

Received: November 2, 2020

Accepted: December 7, $2020 \quad$ Online Published: December 18, 2020

doi:10.5539/ibr.v14n1p18

URL: https://doi.org/10.5539/ibr.v14n1p18

\begin{abstract}
This paper examines how to design the sustainable production line in the factories with minimum environmental impacts and maximum social benefits. For modeling, the literature review is carried out first and according to researches and papers, environmental and social factors are extracted and then with using Sustainable Supply Chain Management structure, Integer Linear programming with using binary variables and Multi Goal Programming, to design an overall sustainable and environmentally friendly production line model with the most positive social effect.
\end{abstract}

Keywords: environmental impacts, integer linear programming model, multi goal programming, social benefits; sustainable production line, sustainable supply chain management

\section{Introduction}

In this paper, in order to review the impacts of factories and production lines on the environment and society, we first review the literature survey which are presented by different researches for using mathematical models. By searching for papers, the papers closest to this issue under investigation were Sustainability papers and the most relevant studies are on supply chain and production line. Then we checked the environmental and social parameters and variables which are used in the different mathematical models. Finally, by using the Multi Goal Programming, Integer linear program sustainable supply chain management structure, we make a model for sustainable production line.

The first time, sustainable supply chain was introduced in 2008. (Seuring, S. \& Muller, M., 2008) found that the concept of integrating environmental, social and economic factors to improve information flow, workflow and environmental protection.

According to searches in papers, most of the environmental and product impact discussions have been found in the Sustainable Supply Chain Management field. Hence the structure used in the production line is based on Sustainability and using SSCM structures.

In this paper, we first review the thematic literature on the three categories of SSCM and the mathematical models solved in SSCM, environmental factors and social factors, then apply the modeling used for the production line based on the literature review and modeling experiences in other fields. Finally, it is summarized and recommendations for further research are given.

In this case, we present a new mathematical model for introducing Sustainable Production line like that some researchers applied responsibilities for environmental protections to the production processes through Cleaner Production. For making our model, we considered the three dimensions of sustainability or triple line model which are social, environmental and economic dimensions.

From a social point of view, we investigate different researches for sustainable supply chain management and social responsibilities and their approach, methods, patterns, etc. for making models. The researchers like: (Alinso,L., Rubino,E.M., Agustina,B., \& Domingo,R., , 2017); (Bai, L., Xie, M., Zhang, Y., Qiao, Q., 2017); (Song, M., Cen, L., Zheng, Z., Fisher, R., Liang, X., Wang,Y., Huisingh, D., 2017); (Tran, T.V., Schnitzer, H., Braunegg, G., \& Lee, H.T., 2017) defined the social responsibilities through Corporate Social Responsibility (CSR) and made a new model. They presented their models with emphasized on the social dimension of sustainability. We used their approach and social variables and parameters which are introduced inn their model, 
in our new model as social responsibility corporation. Most recent researches (Hsueh, 2015); (Tseng, S.C., \& Huang, S.W., 2014); (Tsalis, T.A. \& Evangelinos, K.L., 2019); (Agan,Y., Cemil Kuzey,C., Acar,M. \& Atif,A., 2016) considered social benefits and used mathematical models for making new models.

In terms of environmental protection, the different variables which are effected on the environment, are collected through different researches (Tran, T.V., Schnitzer, H., Braunegg, G., \& Lee, H.T., 2017); (Tsalis, T.A. \& Evangelinos, K.L., 2019); (Tseng, S.C., \& Huang, S.W., 2014). Then we normalize them and use in the new mathematical model.

Research problem is specifically defined by example where the production line undertakes minimize consumed energy and environmental impact, while a factory management wanted minimum costs and also social rules defined social benefits for factories. In this case, that is necessary both parties (production and management) may consider these sustainable dimensions in their efforts. For SPL modeling and implementation, the below steps are done:

- Literature survey including searching papers, Categorizing and Redefine Research Question; The keyword for searching papers are defined as \{SSCM Mathematical Models "or" Mathematical model\} $+\{$ Saving Energy, Environmental Protection, Social Benefits, Cleaner Production, Social Responsibility\};

- Modeling which is used the SSCM structure and Integer programming.

The results show that for implementation SPL, we have different alternative and this model can be as a dynamic program model, because of we should check different alternatives continually and in programming time need to review the production process for re-engineering SPL model.

\section{Literature Review}

\subsection{Sustainable Supply Chain Management}

Sustainability is not a new approach, and the first time (Costanza, R., Patten, B., , 1995) was applied sustainability when he wanted to defining and predicting sustainability. After that (Seuring, S. \& Muller, M., 2008) used sustainability in definition of SCM for introducing an integration between environments, social and economic goals in the industries. Sustainability also known as a triple bottom line (TPL) for designing model. (Devika, K., Jafarian, A. \& Nourbakhsh, V., 2014). The triple line bottom refers to three dimensions of environmental, economic and social. Majority of researches used triple line bottom for making their models either conceptual or mathematical in the field of supply chain management.

\subsection{Sustainable Supply Chain Management Mathematical Models}

For several years, researchers try to define the conceptual designs for this concept, however after 2010, authors designed different mathematical models for solving problems in the scope of SSCM. Mathematical Modeling approaches are applied into SSCM in different ways for making solutions like Multi-Objective programming, integer Linear Programming, fuzzy logic-based Multi Criteria Decision Making (MCDM), Data Envelopment Analysis (DEA), Game Theory, Analysis of Variance (ANOVA), and Goal Programming and so on which is shown in the below table.

Table 1. List of mathematical models which are used by researchers for SSCM

\begin{tabular}{|c|c|c|c|}
\hline ROW & $\begin{array}{l}\text { Mathematical } \\
\text { Model }\end{array}$ & Author(s) & Subject(s) \\
\hline 1 & $\begin{array}{l}\text { Integer Linear } \\
\text { Programming } \\
\text { (ILP) model }\end{array}$ & $\begin{array}{l}\text { (Arampantzi,C., \& Minis., I., 2017) } \\
\text { (Devika, K., Jafarian, A. \& } \\
\text { Nourbakhsh, V., 2014) } \\
\text { (Tran, T.V., Schnitzer, H., } \\
\text { Braunegg, G., \& Lee, H.T., 2017) } \\
\text { (Aktin,T. \& Gergin,z., 2016) }\end{array}$ & $\begin{array}{l}\text { designing sustainable supply chain networks } \\
\text { Designing a sustainable closed-loop supply chain } \\
\text { network } \\
\text { mathematical model for selecting alternatives in } \\
\text { cleaner production programs } \\
\text { Mathematical modelling of sustainable procurement } \\
\text { strategies }\end{array}$ \\
\hline 2 & $\begin{array}{c}\text { DEA } \\
\text { relope Analysis) }\end{array}$ & $\begin{array}{l}\text { (Tajbakhsh, A. \& Hassini, E., 2015). } \\
\text { (Hatami-Marbini, A., Agrell, J., } \\
\text { Tavana, M. \& Khoshnevis, P., 2017) } \\
\text { (Yousefi, S., Soltani, R., Farzipoor, } \\
\text { S.R., Pishvaee, M.S., 2017) } \\
\text { (Lee, T. \& Nam, H., 2016) } \\
\text { (Motevali, H.S., Torabi, S.A., }\end{array}$ & $\begin{array}{l}\text { Evaluate sustainability in SSCM } \\
\text { Sustainable sourcing, Evaluation of efficiency and } \\
\text { effectiveness } \\
\text { Evaluation of SSCM } \\
\text { Impact of Individual and Organizational }\end{array}$ \\
\hline
\end{tabular}




\begin{tabular}{|c|c|c|c|}
\hline & & $\begin{array}{l}\text { Ghasemi, R., 2016) } \\
\text { (Badiezadeh, T., Farzipoor, S.R., \& } \\
\text { Samavati, T., 2017) } \\
\text { (Boudaghi, E. \& Farzipoor S.R., } \\
\text { 2018) } \\
\text { (Ji,x., Wu,J., \& Zhu, Q., 2016) } \\
\text { (Izadikhah, M., Farzipoor S.R., } \\
\text { Ahmadi, K., 2017) }\end{array}$ & $\begin{array}{l}\text { sustainable supply chain networks } \\
\text { Assessing sustainability of supply chains } \\
\text { Predicting group membership of suppliers in } \\
\text { sustainable supply chain } \\
\text { Eco-design of transportation in SSCM } \\
\text { Assessment of sustainability of suppliers in volume } \\
\text { discount context }\end{array}$ \\
\hline 3 & $\begin{array}{l}\text { AHP and Fuzzy } \\
\text { Method }\end{array}$ & $\begin{array}{l}\text { (Luthra, S., Govindan, K., Kannan, } \\
\text { D., Mangla, S.K., \& Garg, C.P., } \\
\text { 2017) } \\
\text { (Mathivathanan, D., Govindan, K., } \\
\text { \& Noorul Haq, A., 2017) } \\
\text { (Aktin,T. \& Gergin,Z., 2016) } \\
\text { (Awasthi, A., Govindan,K., \& Gold, } \\
\text { S., 2018) } \\
\text { (Azimifard, A., Moosavirad, S.H., \& } \\
\text { Ariafar, S., 2018) } \\
\text { (Govindan, K., Shankar, M. \& } \\
\text { Devika, K., 2018) } \\
\text { (Allaoui,H., Guo,Y., Choudhary,A., } \\
\text { \& Bloemho-Rawaard,J.M., } \\
\text { 2017369-384) }\end{array}$ & $\begin{array}{l}\text { Integrated framework for sustainable supplier } \\
\text { selection and evaluation in supply chains } \\
\text { Exploring the impact of dynamic capabilities on } \\
\text { sustainable supply chain firm's performance } \\
\text { Sustainable procurement strategies } \\
\text { Multi-tier sustainable global supplier selection } \\
\text { Selecting sustainable supplier } \\
\text { Supplier selection } \\
\text { Sustainable agro-food supply chain design }\end{array}$ \\
\hline 4 & Game Theory & $\begin{array}{l}\text { (Raj, A., Biswas, I. \& Srivastava, } \\
\text { S.K., 2018) } \\
\text { (Zhu, W. \& He, Y., 2017) }\end{array}$ & $\begin{array}{l}\text { Designing supply contracts for the sustainable supply } \\
\text { chain } \\
\text { Green product design in supply chains under } \\
\text { competition }\end{array}$ \\
\hline 5 & $\begin{array}{l}\text { Analysis } \\
\text { Variance } \\
\text { (ANOVA) }\end{array}$ & $\begin{array}{l}\text { (Popovic, T., Barbosa-Poyoa, A., } \\
\text { Kraslawski, A. \& Carvalho, A., } \\
\text { 2018) }\end{array}$ & Social sustainability assessment of supply chains \\
\hline
\end{tabular}

Given the question of whether designing and modeling the optimal production line is the least harmful to the environment and the most beneficial to the social, a review of the subject literature has been conducted. By looking at previous research and papers, we have come to the category of Sustainability and SSCM, and the researchers have identified environmental factors as variables or parameters in these two cases.

\subsection{Sustainable Production Line}

Supply chain management has different modules which are including Marketing Designing, Supplier Selection, Purchasing and Procurement, Manufacturing and Production, Logistic and transportation, etc. There are limited researches for Sustainable production line which are designed mathematical model. Sustainable production module in SCM refers to different process of manufacturing including designing, supplying raw material, manufacturing, packing and marking and so on. (Alinso,L., Rubino,E.M., Agustina,B., \& Domingo,R., , 2017). The cleaner production program is a methodology and useful model for designing the environmental dimension of sustainable production line (Tran, T.V., Schnitzer, H., Braunegg, G., \& Lee, H.T., 2017). The sustainable production line focus on the process of production and manufacturing which should meet the environmental and social requirement.

\subsection{Corporate Social Responsibility}

Nowadays social responsibility is a common requirement for all factories. There are a huge scholar researches for social responsibility including corporate social responsibility model (Tsalis, T.A. \& Evangelinos, K.L., 2019) (Govindan, K., Shankar, M. \& Devika, K. , 2018). The corporate social responsibility (CSR) is defined as a concept of management which is used by companies and factories for integration of environmental and social concerns in the process and system design and their interactions(Motevali, H.S., Torabi, S.A., Ghasemi, R., 2016). The purpose of CSR is that the companies and factories involve is social responsibility and philanthropic causes which increase the social benefits. CSR highlight the social benefit enablers, and we use this approach for designing our model.

Our aim in this research is to make a mathematical model with using cleaner production and social benefits in the supply chain. The Goal of social benefits are through the lens of two kind of parameters that we considered: negative social impact and positive social impact. (We emphasis on the environmental and social parameters which are related to Sustainable Supply Chain Management). Finally, in this paper we explore how theory from the field of Cleaner Production Program and SSCM can apply for building a mathematical model and, find a solution for implementation the Sustainable Production Line. 


\section{Modeling}

As presented in the Introduction, this paper presents a new method for implementation Sustainable Production Line. We used the Sustainable Supply Chain Management structure as the main structure for making model and used Goal Programming through applying the integer linear program for developing an optimal mathematical model. It is clearly that every production line has different process and if we want to make a model, first we need to define process map of the production line.

In the initial literature review, Goal Programming is an effective decision support tool for alternative selection. (Jones, D.F. \& Tamiz, M., 2002) said that Goal Programming has a widely field in the different researches such as engineering, academic management, agricultural management, energy planning, production planning, and green environmental management.

As per our searching, below papers were found that present a goal-programming methodology related to SSCM, $\mathrm{CP}$ and CSR. Table 2 presents the papers which are used mathematical models for solving SCM problems and achieving the best solution for environment protection and social benefit.

Table 2. Table of papers which are used goal- programming methodology in the mathematical models for SSCM, Cleaner Production and CSR

\begin{tabular}{|c|c|c|}
\hline $\begin{array}{l}\mathbf{R} \\
\mathbf{O} \\
\mathbf{W}\end{array}$ & Author & $\begin{array}{l}\text { Implemented Model/ } \\
\text { Mathematical Model }\end{array}$ \\
\hline 1 & $\begin{array}{l}\text { (Tseng, S.C., \& Huang, S.W., } \\
\text { 2014) }\end{array}$ & Goal programming, linear model \\
\hline 2 & $\begin{array}{l}\text { (Devika, K., Jafarian, A. \& } \\
\text { Nourbakhsh, V., 2014) }\end{array}$ & $\begin{array}{l}\text { Continuous approximation CA, Stochastic mixed integer programming SMIP, } \\
\text { Fuzzy mixed integer programming FMIP, Mixed integer nonlinear } \\
\text { programming MINLP, Mixed integer linear programming }\end{array}$ \\
\hline 3 & (Aktin,T. \& Gergin,z., 2016) & Mixed integer linear programming models, AHP preferences \\
\hline 44 & $\begin{array}{l}\text { (Arampantzi,C., \& Minis., I., } \\
\text { 2017) }\end{array}$ & $\begin{array}{l}\text { Multi-objective supply chain network } \\
\text { Multi-objective Mixed Integer Linear Programming (MMILP) model }\end{array}$ \\
\hline 55 & $\begin{array}{l}\text { (Hatami-Marbini, A., Agrell, } \\
\text { J., Tavana, M. \& Khoshnevis, } \\
\text { P., 2017) }\end{array}$ & $\begin{array}{l}\text { Data envelopment analysis, Fuzzy mathematical programming, } \\
\text { Lexicographic multi-objective linear programming, Fuzzy targets, } \\
\text { Super-efficiency }\end{array}$ \\
\hline 66 & (Zhou, G., \& Zhang, Y., 2017) & mixed Linear Programming \\
\hline 77 & $\begin{array}{l}\text { (Gred, J.H. \& Brandenburg, } \\
\text { M., 2018) }\end{array}$ & $\begin{array}{l}\text { A hierarchical decision support tool is presented that combines a } \\
\text { deterministic } \\
\text { linear programming model and an aggregate stochastic queuing network } \\
\text { model }\end{array}$ \\
\hline 88 & $\begin{array}{l}\text { (Sazvar, Z., Rahmani, M. \& } \\
\text { Govindan, K., 2018) }\end{array}$ & $\begin{array}{l}\text { Multi-objective linear mathematical model, linear model and Pareto solutions, } \\
\text { Augmented 3-constraint (AUGMECON) method }\end{array}$ \\
\hline 99 & $\begin{array}{l}\text { (Raj, A., } \quad \text { Biswas, } \\
\text { Srivastava, S.K., 2018) }\end{array}$ & Two-stage Stackelberg game-theoretic approach, linear two-part tariff (LTT) \\
\hline
\end{tabular}

\subsection{Problem Definition}

The research question is how to design a model for production processes that has the least environmental detrimental effect, benefits the community most, and costs the least.

To cope with this challenge, describe in the introduction, this paper suggest an optimization mathematical model based on goal programming into "cleaner production methodology", "Social Benefits" and "management of costs" as follows:

First, we define Assumptions, Decision variables and parameters. Then, model developing including objective functions and constraints.

\subsection{Definition of the Assumptions, Decision Variables}

This model is based on production process and for improving the production processes and making a sustainable production line, first we define all the process of production line, then we consider different alternatives of every processes. 


\subsubsection{Production Processes}

- Assuming production line has different processes $(\mathrm{Pi}, \mathrm{i}=1,2, \ldots \mathrm{n})$ and every processes have different alternatives (xij, $\mathrm{j}=1,2, \ldots \mathrm{mi}$ ) for performing, we propose a model to find an optimum selections of alternatives which bring the best results for our model.

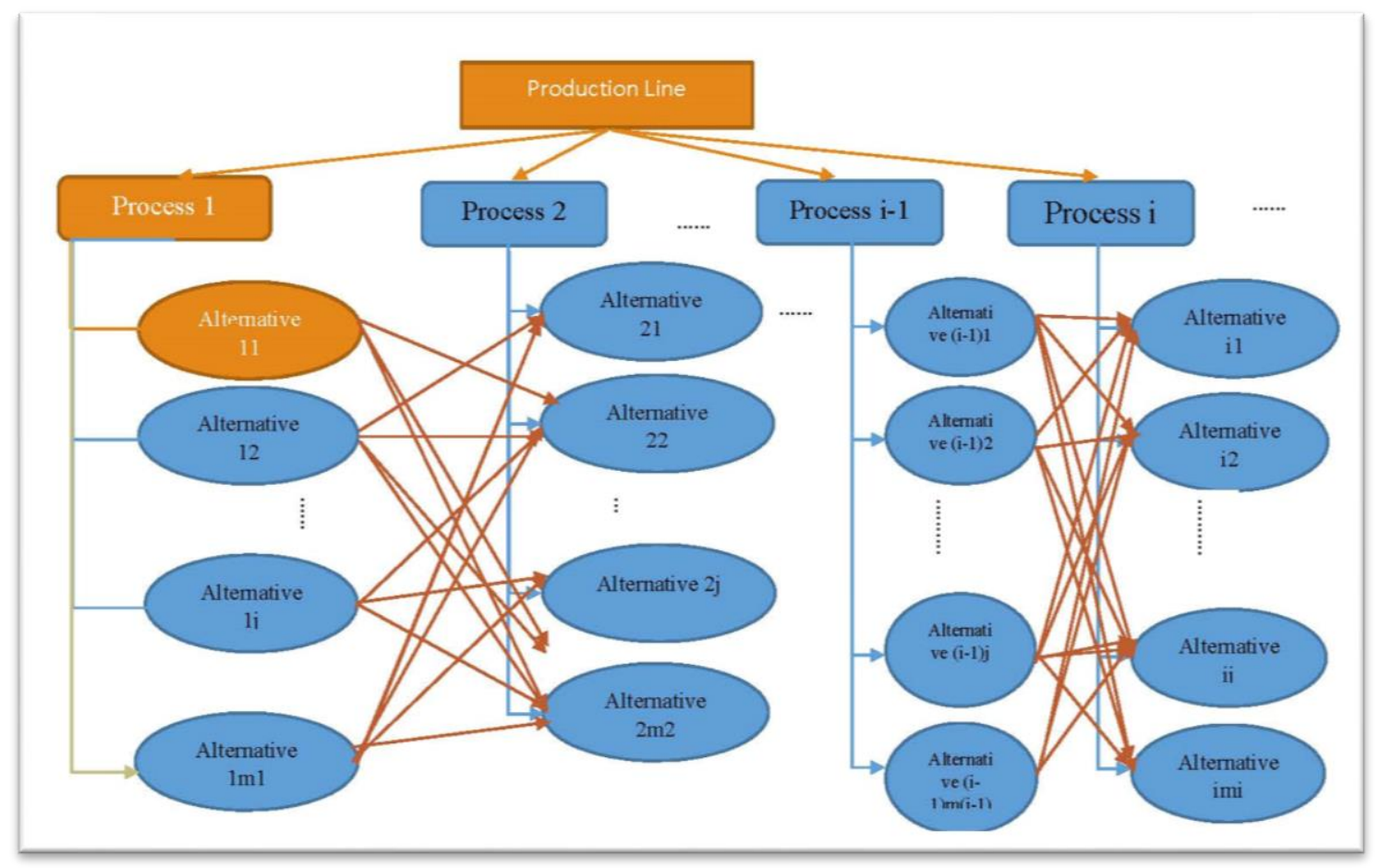

Figure 1. The diagram of processes and alternatives for production line

- We consider $n$ process for production in SPL modeling and each process has $m_{i}$ alternatives for doing process. It is clearly that if one alternative of process "i" is chosen, the other alternatives will be cancelled and it means that we define the variables for alternatives as $\mathrm{x}_{\mathrm{ij}}=1$ (if selected) or 0 (if not selected). Table 3 presents the variables matrix for processes alternatives. On the other hand, we define " $\mathrm{X}_{\mathrm{io}}$ " as baseline production line processes of $\mathrm{Pi}$ (without improvement);

- Every alternatives of process have own costs, environmental impact and social impact. We calculate costs, environmental impact and social impact of every alternatives of processes by using the models that propose by some researchers like Cleaner Production for environmental impact, SCR for social impact and using Accounting models for costs. Then, we make a model for choosing the optimum solution for combination of processes and make a SPL (Sustainable Production Line.) Table 3 shows the decision variable matrix for different Alternatives of production processes. Every column shows the alternatives for every process.

Table 3. Decision Variable matrix for Processes Alternatives

\begin{tabular}{|c|c|c|c|c|c|}
\hline $\mathrm{x}_{11}$ & $\mathrm{X}_{21}$ & $\ldots$ & $\mathrm{x}_{\mathrm{i} 1}$ & $\ldots$ & $x_{n 1}$ \\
\hline $\mathrm{x}_{12}$ & $\mathrm{x}_{22}$ & $\ldots$ & $\mathrm{x}_{\mathrm{i} 2}$ & $\ldots$ & $\mathrm{x}_{\mathrm{n} 2}$ \\
\hline$\ldots$ & $\ldots$ & $\ldots$ & $\ldots$ & $\ldots$ & $\ldots$ \\
\hline $\mathrm{x}_{1 \mathrm{i}}$ & $\ldots$ & $\ldots$ & $\mathrm{x}_{\mathrm{ij}}$ & $\ldots$ & $\mathrm{x}_{\mathrm{ni}}$ \\
\hline$\ldots$ & $\ldots$ & $\ldots$ & $\ldots$ & $\ldots$ & $\ldots$ \\
\hline $\mathrm{x}_{1 \mathrm{n}}$ & $\mathrm{X}_{2 \mathrm{n}}$ & $\ldots$ & $\mathrm{X}_{\mathrm{imi}}$ & $\ldots$ & $\mathrm{X}_{\mathrm{nmn}}$ \\
\hline
\end{tabular}

\subsubsection{Environmental Impact}

Every alternative of the process has own environmental impact which can be seen in the environment impact matrix as per below table. 
Table 4. The matrix of Environmental impact reduction variables

\begin{tabular}{|c|c|c|c|c|c|}
\hline $\mathbf{E}_{11}$ & $\mathbf{E}_{21}$ & $\ldots$ & $\mathbf{E}_{\mathrm{i} 1}$ & $\ldots$ & $E_{n 1}$ \\
\hline$E_{12}$ & $\mathrm{E}_{22}$ & $\ldots$ & $\mathrm{E}_{\mathrm{i} 2}$ & $\ldots$ & $\mathrm{E}_{\mathrm{n} 2}$ \\
\hline .... & $\ldots$ & $\ldots$ & $\ldots$ & $\ldots$ & $\ldots$ \\
\hline $\mathbf{E}_{1 \mathrm{j}}$ & $\ldots$ & $\ldots$ & $\mathrm{E}_{\mathrm{ij}}$ & $\ldots$ & $\mathrm{E}_{\mathrm{ni}}$ \\
\hline$\ldots$ & $\ldots$ & $\ldots$ & $\ldots$ & $\ldots$ & $\ldots$ \\
\hline$E_{1 n}$ & $E_{2 n}$ & $\ldots$ & $\mathrm{E}_{\mathrm{imi}}$ & $\ldots$ & $\mathrm{E}_{\mathrm{nmn}}$ \\
\hline
\end{tabular}

$E_{i j}$ : environmental impact variable of alternative $X_{i j}$

For calculating the environmental impact, we have different index and parameters. We use the sustainability development index (2019) and the summary of parameters which are suggested by different researchers through cleaner production planning researches (Table 5).

Table 5. List of Environmental Impact Variables

\begin{tabular}{|c|c|c|c|c|}
\hline Ref. & Material & Parameter & Measurement way & Symbol \\
\hline $\begin{array}{l}\text { Tsalis.T.A and Evangelinos.K.I (2019); Tran.T.V, } \\
\text { Schnitzer.H, Braunegg.G \& Lee.H.T.(2017) }\end{array}$ & Energy & $\begin{array}{l}\text { Energy Use } \\
\text { Electricity } \\
\text { Heating amount } \\
\text { Cooling amount }\end{array}$ & $\begin{array}{l}\text { MJ/ } \\
\text { Quantity /year }\end{array}$ & $\mathrm{E}_{\text {energy }}$ \\
\hline $\begin{array}{l}\text { Tsalis.T.A and Evangelinos.K.I (2019); Tran.T.V, } \\
\text { Schnitzer.H, } \\
\text { Braunegg.G\&Lee.H.T.(2017);Aktin.T\&Gergin.Z.(20 } \\
\text { 16), }\end{array}$ & Water & Water Use & $\mathrm{m}^{3}$ water/Quantity/year & $\mathrm{E}_{\text {water }}$ \\
\hline $\begin{array}{l}\text { Tsalis.T.A and Evangelinos.K.I (2019); Tran.T.V, } \\
\text { Schnitzer.H, } \\
\text { Braunegg.G\&Lee.H.T.(2017);Aktin.T\&Gergin.Z.(20 } \\
\text { 16), }\end{array}$ & Emission & $\begin{array}{l}\mathrm{CO}_{2} \\
\mathrm{CH}_{4} \\
\mathrm{~N} 2 \mathrm{O} \\
\mathrm{HFCs} \\
\mathrm{PFCs} \\
\mathrm{NF} 3\end{array}$ & $\begin{array}{l}\text { TonCO2/Quantity/year } \\
\text { Ton } \mathrm{CH}_{4} / \text { Quantity/year } \\
\text { TonN2O/Quantity/year } \\
\text { TonHFCs/Quantity/yea } \\
\mathrm{r} \\
\text { Ton } \\
\text { PFCs/Quantity/year } \\
\text { Ton NF3/Quantity/year }\end{array}$ & $\begin{array}{l}\mathrm{E}_{\mathrm{C} 22} \\
\mathrm{E}_{\mathrm{CH} 4} \\
\mathrm{E}_{\mathrm{N} 20} \\
\mathrm{E}_{\mathrm{HFCs}} \\
\mathrm{E}_{\mathrm{PFCs}} \\
\mathrm{E}_{\mathrm{NF3} 3}\end{array}$ \\
\hline $\begin{array}{l}\text { Tran.T.V, } \\
\text { Braunegg.G\&Lee.H.T.(2017); Motevali Haghighi.S, } \\
\text { Torabi.S.A , Ghasemi.R (2016); Awasthi.A , } \\
\text { Govindan.K \& Gold.S. (2018), }\end{array}$ & Waste & $\begin{array}{l}\text { Solid Waste } \\
\text { Hazardous waste }\end{array}$ & $\begin{array}{l}\mathrm{kg} / \text { Quantity/year } \\
\mathrm{kg} / \text { Quantity/year }\end{array}$ & $\begin{array}{l}\mathrm{E}_{\mathrm{sw}} \\
\mathrm{E}_{\mathrm{hw}}\end{array}$ \\
\hline $\begin{array}{l}\text { Tsalis.T.A and Evangelinos.K.I (2019); Awasthi.A, } \\
\text { Govindan.K \& Gold.S. (2018), }\end{array}$ & $\begin{array}{l}\text { Bio } \\
\text { Diversity }\end{array}$ & $\begin{array}{l}\text { size of } \\
\text { operational site } \\
\text { in km2 } \\
\text { impact on local } \\
\text { species ratio of } \\
\text { protected habits }\end{array}$ & & $\mathrm{E}_{\text {bio }}$ \\
\hline
\end{tabular}

For every alternatives of process, we have above parameters. It means that for Calculating Eij of alternative $\mathrm{j}$ of process $\mathrm{i}$, referring to table (4), we have different amount of environmental impact like $\mathrm{E}(\mathrm{P})_{\mathrm{ij}}$ :

$$
\text { Equation 1: } E(P)_{i j}=\left\{E_{(e n e r g y) i j}, E_{(w a t e r) i j}, E_{(c o 2) i j}, E_{(C H 4) i j}, E_{(N 2 O) i j}, E_{(H F C s) i j}, E_{(P F C S) i j}, E_{(N P 3) i j}, E_{(s w) i j}, E_{(H w) i j}, E_{(b i o) i j}\right\} \text {. }
$$

For Calculating $\mathrm{E}_{\mathrm{ij}}$, we have to normalize different environmental impact variables and then we can calculate the total environmental impact of alternative $\mathrm{j}$ process $\mathrm{i}$. We use Normal Distribution for normalizing the environmental parameters. The formula that we use is:

$$
\begin{gathered}
\text { Equation 2: } n=\frac{x-\mu}{\delta} \quad \text { where: }=\frac{\sum_{i=1}^{n} X i}{n}, \delta=\sqrt[2]{\frac{x i-\mu}{\frac{\sum_{i=1}^{n}(X i-\mu)^{2}}{n}}} \\
\text { Equation 3: } n_{i j(P)}=\left(\frac{E(P) i j-\frac{\sum_{i} \Sigma_{j} E(P) i j}{m * n}}{\sqrt{\frac{\sum_{i} \Sigma_{j}\left(E(P) i j-\frac{\sum_{i} \Sigma_{j} E(P) i j}{m * n}\right)^{2}}{m * n}}}\right),
\end{gathered}
$$


with considering weight of every environmental parameter, we can calculate the $\mathrm{E}_{\mathrm{ij}}$ as per below equation:

$$
\text { Equation 4: } E_{i j}=\sum n i j(P)=\sum\left(\frac{E(P) i j-\frac{\sum_{i} \Sigma_{j} E(P) i j}{m * n}}{\sqrt{\frac{\left(\sum_{i} \Sigma_{j}\left(E(P) i j-\frac{\sum_{i} \Sigma_{j} E(P) i j}{m * n}\right)^{2}\right.}{m * n}}}\right)
$$

The result of Equations 2, 3 and 4 is :

Equation

$E i j=\frac{E(\text { Energy }) i j-\frac{\sum_{i} \sum_{j} E(\text { Energy }) i j}{m * n}}{\sqrt{\frac{\left(\sum_{i} \sum_{j}\left(E(\text { Energy }) i j-\frac{\sum_{i} \sum_{j} E(\text { Energy }) i j}{m * n}\right)^{2}\right.}{m * n}}}+\frac{E(\text { Water }) i j-\frac{\sum_{i} \sum_{j} E(\text { water }) i j}{m * n}}{\sqrt{\frac{\left(\sum_{i} \sum_{j}\left(E(\text { water }) i j-\frac{\sum_{i} \sum_{j} E(\text { water }) i j}{m * n}\right)^{2}\right.}{m * n}}}+\frac{E(C O 2) i j-\frac{\sum_{i} \Sigma_{j} E(\mathrm{CO} 2) i j}{m * n}}{\sqrt{\frac{\left(\sum_{i} \sum_{j}\left(E(C O 2) i j-\frac{\sum_{i} \sum_{j} E(C O 2) i j}{m * n}\right)^{2}\right.}{m * n}}}+$

$\frac{E(N 2 O) i j-\frac{\sum_{i} \sum_{j} E(N 2 O) i j}{m * n}}{\sqrt{\frac{\left(\sum_{i} \sum_{j}\left(E(N 2 O) i j-\frac{\sum_{i} \sum_{j} E(N 2 O) i j}{m * n}\right)^{2}\right.}{m * n}}}+\frac{E(H F C s) i j-\frac{\sum_{i} \sum_{j} E(H F C s) i j}{m * n}}{\sqrt{\frac{\left(\sum_{i} \sum_{j}\left(E(H F C s) i j-\frac{\sum_{i} \sum_{j} E(H F C S) i j}{m * n}\right)^{2}\right.}{m * n}}}+\frac{E(P F C S) i j-\frac{\sum_{i} \Sigma_{j} E(P F C s) i j}{m * n}}{\sqrt{\frac{\left(\sum_{i} \sum_{j}\left(E(P F C s) i j-\frac{\sum_{i} \sum_{j} E(P F C s) i j}{m * n}\right)^{2}\right.}{m * n}}}+$

$\frac{E(N P 3) i j-\frac{\sum_{i} \Sigma_{j} E(N P 3) i j}{m * n}}{\sqrt{\frac{\left(\sum_{i} \sum_{j}\left(E(N P 3) i j-\frac{\sum_{i} \Sigma_{j} E(N P 3) i j}{m * n}\right)^{2}\right.}{m * n}}}+\frac{E(S w) i j-\frac{\sum_{i} \Sigma_{j} E(S w) i j}{m * n}}{\sqrt{\frac{\left(\sum_{i} \sum_{j}\left(E(S w) i j-\frac{\sum_{i} \sum_{j} E(S w) i j}{m * n}\right)^{2}\right.}{m * n}}}+\frac{E(H w) i j-\frac{\sum_{i} \sum_{j} E(H w) i j}{m * n}}{\sqrt{\frac{\left(\sum_{i} \sum_{j}\left(E(H w) i j-\frac{\sum_{i} \Sigma_{j} E(H w) i j}{m * n}\right)^{2}\right.}{m * n}}}+\frac{E(b i o) i j-\frac{\sum_{i} \Sigma_{j} E(b i o) i j}{m * n}}{\sqrt{\frac{\left(\sum_{i} \sum_{j}\left(E(b i o) i j-\frac{\sum_{i} \sum_{j} E(b i o) i j}{m * n}\right)^{2}\right.}{m * n}}}$

\subsubsection{Economic Parameters}

Assuming the development SPL economic variables as per below:

○ $\quad \mathrm{D}_{\mathrm{y}}$ : Annual Demand for production;

○ $\mathrm{T}_{\mathrm{ij}}$ : time duration of $\mathrm{X}_{\mathrm{ij}}$;

○ $\mathrm{C}_{\mathrm{pij}}$ : total cost for process of $\mathrm{X}_{\mathrm{ij}}$ including direct and indirect costs;

○ $\mathrm{C}_{\mathrm{Eij}}$ : total cost for improvement or reduction for $\mathrm{X}_{\mathrm{ij}}$;

○ $\mathrm{C}_{\mathrm{Sij}}$ : Cost for social impact of $\mathrm{X}_{\mathrm{ij}}$;

○ $\mathrm{Cp}$ : total budget for production line;

○ $\mathrm{C}_{\mathrm{Xij}}$ : total costs of alternative $\mathrm{X}_{\mathrm{ij}}$;

○ $\mathrm{Cc}$ : Cost for purchasing carbon credit for production line according to rules and regulation of government for environmental protection.

Equation 6: $\mathrm{C}_{\mathrm{p}} \leq\left(\sum_{j=1}^{m n} \sum_{i=1}^{n} C p i j * X i j+\sum_{j=1}^{m n} \sum_{i=1}^{n} C E i j * X i j+\sum_{j=1}^{m n} \sum_{i=1}^{n} C s i j * X i j+\mathrm{C}_{\mathrm{c}}\right)$

We have three matrixes for Costs which are Economic Costs $\left(\mathrm{C}_{\mathrm{pij}}\right)$ Matrix, Environmental reduction costs Matrix $\left(\mathrm{C}_{\mathrm{Eij}}\right)$ and Social impact costs $\left(\mathrm{C}_{\mathrm{Sij}}\right)$. We define $\mathrm{C}_{\mathrm{Xij}}$ as per Equation 6.

$$
\text { Equation 7: } \mathrm{C}_{\mathrm{Xij}}=C_{p i j}+C_{E i j}+\mathrm{C}_{\mathrm{Sij}}
$$

We have a cost matrix for processes alternatives as per below table.

\begin{tabular}{|c|c|c|c|c|c|}
\hline$C x_{11}$ & $\mathrm{Cx}_{21}$ & $\ldots$ & $C x_{i 1}$ & $\ldots$ & $C x_{n 1}$ \\
\hline $\mathrm{Cx}_{12}$ & $\mathrm{Cx}_{22}$ & .... & $\mathrm{Cx}_{\mathrm{i} 2}$ & .... & $\mathrm{Cx}_{\mathrm{n} 2}$ \\
\hline$\ldots$ & $\ldots$ & $\ldots$ & $\ldots$ & $\ldots$ & $\ldots$ \\
\hline $\mathbf{C} \mathbf{x}_{1 \mathbf{j}}$ & .... & $\ldots$ & $\mathrm{Cx}_{\mathrm{ij}}$ & .... & $\mathrm{Cx}_{\mathrm{ni}}$ \\
\hline$\ldots$ & $\ldots$ & $\ldots$ & $\ldots$ & $\ldots$ & $\ldots$ \\
\hline $\mathbf{C} \mathbf{x}_{1 \mathrm{n}}$ & $\mathrm{Cx}_{2 \mathrm{n}}$ & $\ldots$ & $C x_{\text {imi }}$ & $\ldots$ & $\mathrm{Cx}_{\mathrm{nmn}}$ \\
\hline
\end{tabular}

Table 6. Cost Matrix for Alternatives of Processes 


\subsubsection{Social Parameters}

Nowadays one of the important responsibilities of factories is social responsibility. For designing social responsibilities systems in the factories and implementation and measurement, researchers proposed different models. We study these models and most important is the parameters and criteria for measurements and modeling the social responsibilities. Different researchers offer various parameters such as decent work conditions, Human rights and abuse human rights, personal training, health and safety, rewards and punishments criteria and so on. (Table 7). According to the research results and literature review, we define two kind of social impacts: positive impacts and negative impacts.

Table 7. List of social responsibilities parameters for factories

\begin{tabular}{|c|c|c|c|c|c|}
\hline Ref. & Parameter & Unit & Symbol & Measurement way & Social Impact \\
\hline $\begin{array}{l}\text { (Awasthi, A., Govindan,K., } \\
\text { \& Gold, S., 2018); } \\
\text { (Govindan, K., Shankar, M. } \\
\text { \& Devika, K., 2018) }\end{array}$ & Labor Practices & Man-Hours/year & $\mathrm{S}_{\mathrm{lr}}$ & $\begin{array}{l}\text { Total Hours of } \\
\text { practices per year }\end{array}$ & Positive \\
\hline $\begin{array}{l}\text { (Awasthi, A., Govindan,K., } \\
\text { \& Gold, S., 2018); } \\
\text { (Govindan, K., Shankar, M. } \\
\text { \& Devika, K., 2018) }\end{array}$ & $\begin{array}{l}\text { Decent work } \\
\text { Conditions } \\
\text { Flexible working }\end{array}$ & $\begin{array}{l}\text { Satisfaction } \\
\text { Score }\end{array}$ & $\mathrm{S}_{\mathrm{df}}$ & $\begin{array}{l}\text { Worker interview, } \\
\text { questionnaire and } \\
\text { survey }\end{array}$ & Positive \\
\hline $\begin{array}{l}\text { (Awasthi, A., Govindan,K., } \\
\text { \& Gold, S., 2018); } \\
\text { (Govindan, K., Shankar, M. } \\
\text { \& Devika, K., 2018) }\end{array}$ & $\begin{array}{l}\text { Human Rights/ } \\
\text { Worker rights } \\
\text { Abuse of human } \\
\text { right/ } \\
\text { Human rights of an } \\
\text { individual }\end{array}$ & $\begin{array}{l}\text { Number } \\
\text { complains }\end{array}$ & $\begin{array}{l}\mathrm{S}_{\mathrm{hr}} \\
\mathrm{S}_{\mathrm{ah}}\end{array}$ & $\begin{array}{l}\text { Survey } \\
\text { HR Data }\end{array}$ & $\begin{array}{l}\text { Positive } \\
\text { Negative }\end{array}$ \\
\hline $\begin{array}{l}\text { (Tsalis, T.A. \& Evangelinos, } \\
\text { K.L., 2019) (Govindan, K., } \\
\text { Shankar, M. \& Devika, K. , } \\
\text { 2018) (Arampantzi,C., \& } \\
\text { Minis., I., 2017) }\end{array}$ & $\begin{array}{l}\text { Employment } \\
\text { Health and Safety }\end{array}$ & $\begin{array}{l}\text { Number of } \\
\text { accidents / Year }\end{array}$ & $\mathrm{SS}_{\mathrm{eh}}$ & $\begin{array}{lr}\text { Survey } & \text { and } \\
\text { Human } & \text { resource } \\
\text { data } & \end{array}$ & Negative \\
\hline $\begin{array}{l}\text { (Tsalis, T.A. \& Evangelinos, } \\
\text { K.L., 2019) (Govindan, K., } \\
\text { Shankar, M. \& Devika, K. , } \\
\text { 2018) }\end{array}$ & Training & $\begin{array}{l}\text { Number of } \\
\text { Human who } \\
\text { trained / year }\end{array}$ & $\mathrm{S}_{\mathrm{tr}}$ & $\begin{array}{lr}\text { Survey } & \text { and } \\
\text { Human } & \text { resource } \\
\text { data } & \end{array}$ & Positive \\
\hline $\begin{array}{l}\text { (Tsalis, T.A. \& Evangelinos, } \\
\text { K.L., 2019) }\end{array}$ & $\begin{array}{l}\text { Voice of } \\
\text { Customers }\end{array}$ & $\begin{array}{l}\text { Customer } \\
\text { Satisfaction } \\
\text { score and } \\
\text { Complains }\end{array}$ & $\begin{array}{l}\mathrm{S}_{\mathrm{cs}} \\
\mathrm{S}_{\mathrm{cp}}\end{array}$ & CSR Data & $\begin{array}{l}\text { Positive } \\
\text { Negative }\end{array}$ \\
\hline $\begin{array}{l}\text { (Tajbakhsh, A. \& Hassini, } \\
\text { E., 2015) }\end{array}$ & $\begin{array}{l}\text { average reputation } \\
\text { factor }\end{array}$ & Score & $\mathrm{S}_{\mathrm{ar}}$ & Survey & positive \\
\hline $\begin{array}{l}\text { (Govindan, K., Shankar, M. } \\
\text { \& Devika, K., 2018) }\end{array}$ & $\begin{array}{ll}\text { Equity } & \text { Labor } \\
\text { Sources } & \end{array}$ & Score & $\mathrm{S}_{\mathrm{El}}$ & Survey & positive \\
\hline $\begin{array}{l}\text { (Govindan, K., Shankar, M. } \\
\text { \& Devika, K., 2018) }\end{array}$ & $\begin{array}{l}\text { Rewards for ethical } \\
\text { behavior } \\
\text { Penalties and } \\
\text { punishments for } \\
\text { unethical behavior }\end{array}$ & $\begin{array}{l}\text { The quantity of } \\
\text { rewards } \\
\text { The quantity of } \\
\text { punishment }\end{array}$ & $\mathrm{S}_{\mathrm{pu}}$ & HR Data & $\begin{array}{l}\text { Positive } \\
\text { Negative }\end{array}$ \\
\hline $\begin{array}{l}\text { (Tsalis, T.A. \& Evangelinos, } \\
\text { K.L., 2019) }\end{array}$ & Job Opportunities & $\begin{array}{l}\text { The total } \\
\text { quantity of Job } \\
\text { Opportunities }\end{array}$ & $\mathrm{S}_{\mathrm{jb}}$ & Production plan & Positive \\
\hline $\begin{array}{l}\text { (Govindan, K., Shankar, M. } \\
\text { \& Devika, K., 2018) }\end{array}$ & $\begin{array}{l}\text { Product } \\
\text { responsibility }\end{array}$ & Score & $\mathrm{S}_{\mathrm{pr}}$ & Survey & Positive \\
\hline (Hsueh, 2015) & $\begin{array}{l}\text { CSR Performance } \\
\text { Level }\end{array}$ & Score & $\mathrm{S}_{\mathrm{csr}}$ & Survey & Positive \\
\hline
\end{tabular}

For normalizing the parameters and using in the model, we use the normal distribution same as Environmental parameters in Equation 3.

Equation $8: S_{(x p)}=$ The collection of positive of social parameters $€\left\{S_{l r}, S_{d f}, S_{h r}, S_{t r}, S_{c s}, S_{a r}, S_{e l}, S_{r w}, S_{p r}, S_{j b}\right.$,

$$
\left.S_{c s r}\right\}
$$


Equation 9: $S_{(x n)}=$ The collection of negative of social parameters $€\left\{S_{a h,}, S_{e h}, S_{c p}, S_{p u}\right\}$

Equation 10: $\quad S_{i j}=$ the summation of normalized positive social parameters minus negative social parameters

$$
=\quad \sum_{x} \frac{S(x p) i j-\frac{\Sigma_{i} \Sigma_{j} S(x p) i j}{m * n}}{\sqrt{\frac{\left(\sum_{i} \Sigma_{j}\left(S(x p) i j-\frac{\sum_{i} \Sigma_{j} S(x p) i j}{m * n}\right)^{2}\right.}{m * n}}}-\sum_{x} \frac{S(x n) i j-\frac{\sum_{i} \Sigma_{j} S(x n) i j}{m * n}}{\sqrt{\frac{\left(\sum_{i} \Sigma_{j}\left(S(x n) i j-\frac{\Sigma_{i} \Sigma_{j} S(x n) i j}{m * n}\right)^{2}\right.}{m * n}}}
$$

With using Equation (8), Equation (9) and Equation (10):

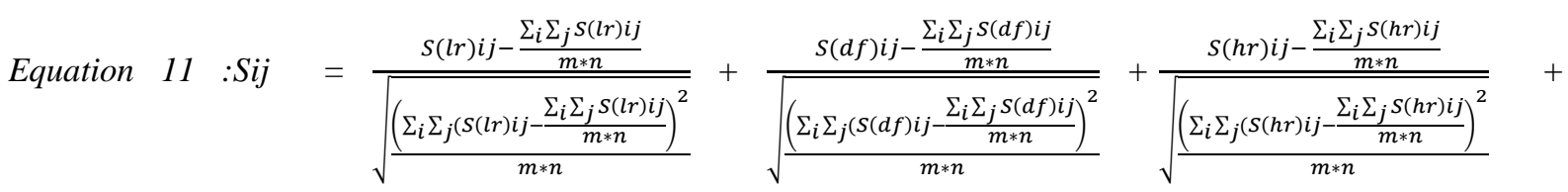

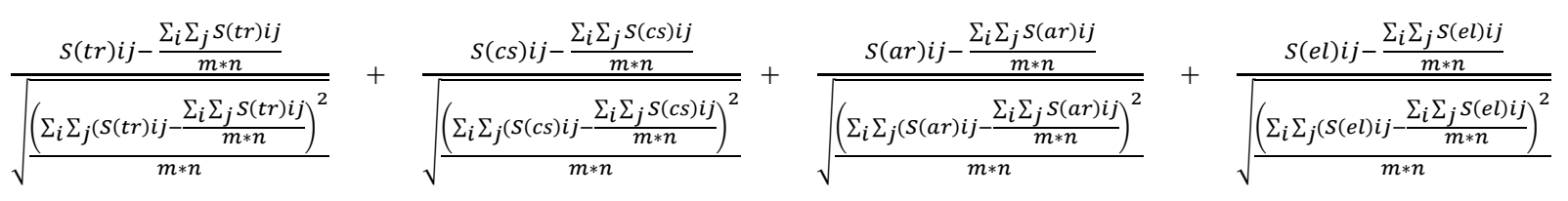

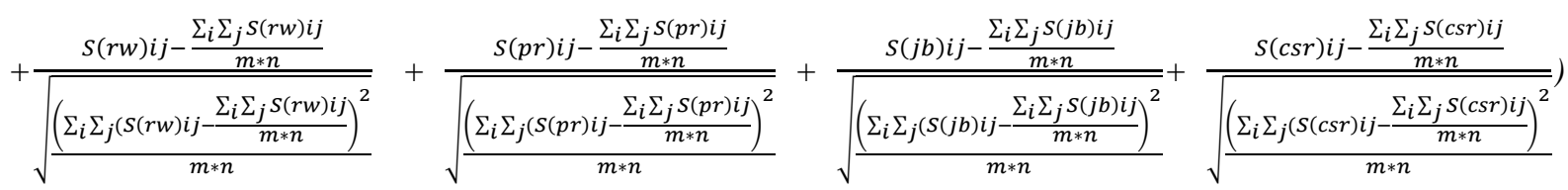

$-\left(\frac{S(a h) i j-\frac{\sum_{i} \Sigma_{j} S(a h) i j}{m * n}}{\sqrt{\frac{\left(\sum_{i} \Sigma_{j}\left(S(a h) i j-\frac{\sum_{i} \Sigma_{j} S(a h) i j}{m * n}\right)^{2}\right.}{m * n}}}+\frac{S(e h) i j-\frac{\sum_{i} \Sigma_{j} S(e h) i j}{m * n}}{\sqrt{\frac{\left(\sum_{i} \Sigma_{j}\left(S(e h) i j-\frac{\sum_{i} \Sigma_{j} S(e h) i j}{m * n}\right)^{2}\right.}{m * n}}}+\frac{S(c p) i j-\frac{\sum_{i} \Sigma_{j} S(c p) i j}{m * n}}{\sqrt{\frac{\left(\sum_{i} \Sigma_{j}\left(S(c p) i j-\frac{\sum_{i} \Sigma_{j} S(c p) i j}{m * n}\right)^{2}\right.}{m * n}}}+\frac{S(p u) i j-\frac{\sum_{i} \Sigma_{j} S(p u) i j}{m * n}}{\sqrt{\frac{\left(\sum_{i} \Sigma_{j}\left(S(p u) i j-\frac{\sum_{i} \Sigma_{j} S(p u) i j}{m * n}\right)^{2}\right.}{m * n}}}\right.$

The result of the Equations 7-11 are a matrix as per below table which is shown the social impacts in every alternatives:

Table 8. The matrix of Social Impacts for different alternatives

\begin{tabular}{|l|l|l|l|l|l|}
\hline $\mathrm{S}_{11}$ & $\mathrm{~S}_{21}$ & $\ldots$. & $\mathrm{S}_{\mathrm{i} 1}$ & $\ldots$ & $\mathrm{S}_{\mathrm{n} 1}$ \\
\hline $\mathrm{S}_{12}$ & $\mathrm{~S}_{22}$ & $\ldots$. & $\mathrm{S}_{\mathrm{i} 2}$ & $\ldots$. & $\mathrm{S}_{\mathrm{n} 2}$ \\
\hline$\ldots$. & $\ldots$. & $\ldots$. & $\ldots$. & $\ldots$. & $\ldots$. \\
\hline $\mathrm{S}_{1 \mathrm{i}}$ & $\ldots$. & $\ldots$. & $\mathrm{S}_{\mathrm{ij}}$ & $\ldots$. & $\mathrm{S}_{\mathrm{ni}}$ \\
\hline$\ldots$ & $\ldots$. & $\ldots$. & $\ldots$. & $\ldots$. & $\ldots$. \\
\hline $\mathrm{S}_{1 \mathrm{n}}$ & $\mathrm{S}_{2 \mathrm{n}}$ & $\ldots$. & $\mathrm{S}_{\mathrm{imi}}$ & $\ldots$. & $\mathrm{S}_{\mathrm{nmn}}$ \\
\hline
\end{tabular}

\subsection{Model Development}

The main issues for the production line is which alternatives for every process should be selected in order to minimum environmental impacts and maximum social benefits with production constraints and budget constraints. The Goals are defined according to research question as per below:

○ Goal 1- Minimize the environmental impact with considering limitation of budget for improvement

○ Goal 2- Minimize the total cost with considering environmental impact objective;

○ Goal 3: Maximize social benefits with considering budget limitations. 


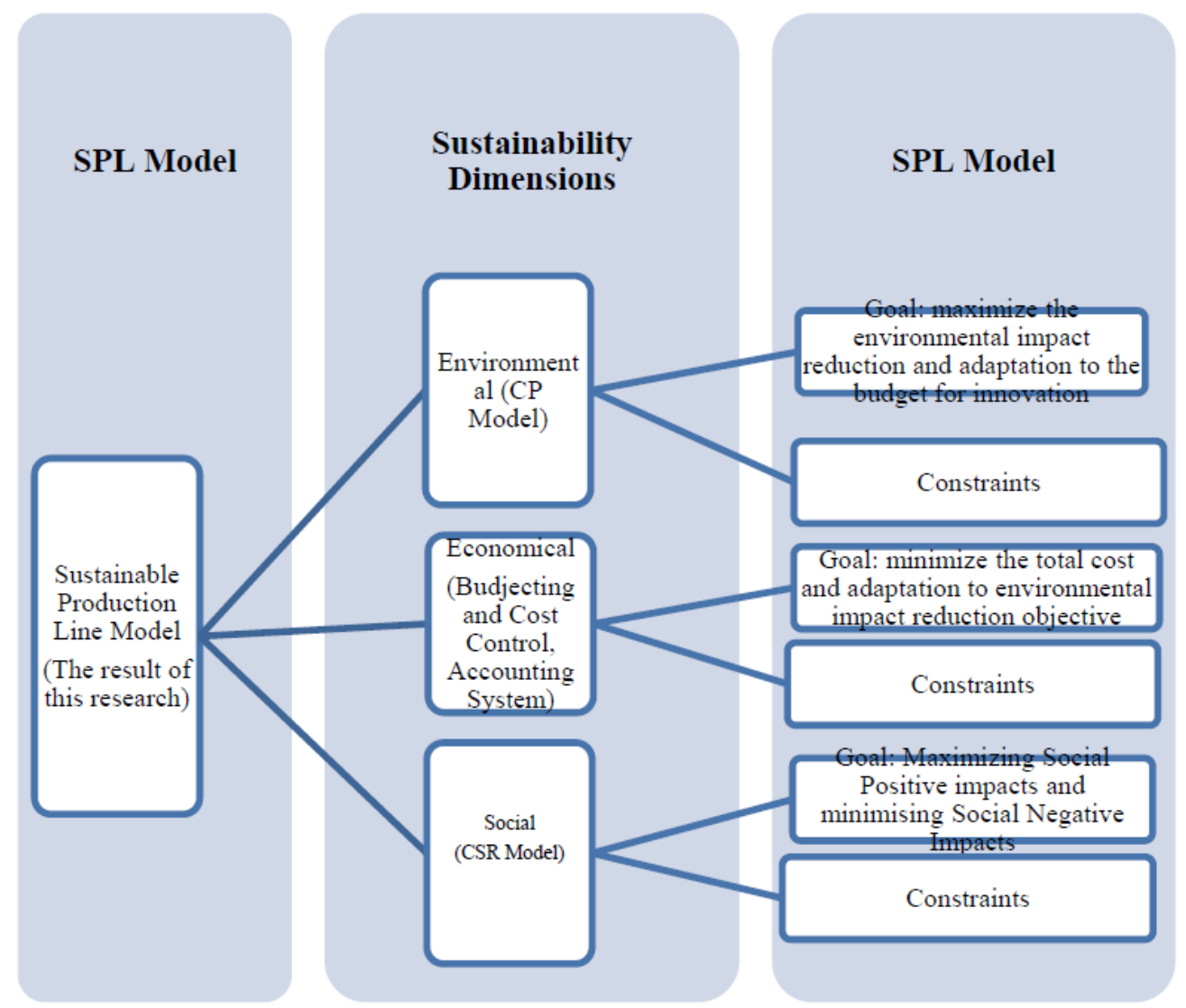

Figure 2. The diagram of Different models combinations for making new Sustainable Production Line model

- Model formulation

The objective functions of the three dimensions are as follows:

\subsubsection{Objective Functions}

- Objective function of Goal 1: The goal is the minimization of the environmental impact. Environmental impact is expressed as in Equation (12).

$$
\text { Equation 12: } \quad G 1=\sum_{i=1}^{n} \sum_{j=0}^{m i} E i j * X i j
$$

- $\quad \mathrm{E}_{\mathrm{ij}}$ : Environmental Parameter of process Pi, $\mathrm{i}=1 \ldots \mathrm{n}$ which are expressed in section 3-2-2;

- $\mathrm{n}$ : total number of processes of the plant;

- $\mathrm{m}_{\mathrm{i}}$ : The number of alternatives for process $\mathrm{i}$.

From Equation (1), the integrated environmental parameter of choosing the best alternatives of processes is expressed as Equation (5):

\section{Equation 13: G1}

$$
=\left(\sum _ { i } \sum _ { j } \left(\frac{E(E n e r g y) i j-\frac{\sum_{i} \Sigma_{j} E(E n e r g y) i j}{m * n}}{\sqrt{\frac{\left(\sum_{i} \Sigma_{j}\left(E(E n e r g y) i j-\frac{\Sigma_{i} \Sigma_{j} E(E n e r g y) i j}{m * n}\right)^{2}\right.}{m * n}}}+\frac{E(\text { Water }) i j-\frac{\sum_{i} \Sigma_{j} E(\text { water }) i j}{m * n}}{\sqrt{\frac{\left(\sum_{i} \Sigma_{j}\left(E(\text { water }) i j-\frac{\Sigma_{i} \Sigma_{j} E(\text { water }) i j}{m * n}\right)^{2}\right.}{m * n}}}+\frac{E(C O 2) i j-\frac{\sum_{i} \Sigma_{j} E(C O 2) i j}{m * n}}{\sqrt{\frac{\left(\sum_{i} \Sigma_{j}\left(E(C O 2) i j-\frac{\Sigma_{i} \Sigma_{j} E(C O 2) i j}{m * n}\right)^{2}\right.}{m * n}}}+\right.\right.
$$




$$
\begin{aligned}
& \frac{E(N 2 O) i j-\frac{\sum_{i} \sum_{j} E(N 2 O) i j}{m * n}}{\sqrt{\frac{\left(\sum_{i} \Sigma_{j}\left(E(N 2 O) i j-\frac{\sum_{i} \Sigma_{j} E(N 2 O) i j}{m * n}\right)^{2}\right.}{m * n}}}+\frac{E(H F C s) i j-\frac{\sum_{i} \sum_{j} E(H F C S) i j}{m * n}}{\sqrt{\frac{\left(\sum_{i} \sum_{j}\left(E(H F C s) i j-\frac{\sum_{i} \sum_{j} E(H F C s) i j}{m * n}\right)^{2}\right.}{m * n}}}+\frac{E(P F C s) i j-\frac{\sum_{i} \Sigma_{j} E(P F C s) i j}{m * n}}{\sqrt{\frac{\left(\sum_{i} \sum_{j}\left(E(P F C s) i j-\frac{\sum_{i} \sum_{j} E(P F C s) i j}{m * n}\right)^{2}\right.}{m * n}}}+ \\
& \frac{E(N P 3) i j-\frac{\sum_{i} \Sigma_{j} E(N P 3) i j}{m * n}}{\sqrt{\frac{\left(\sum_{i} \Sigma_{j}\left(E(N P 3) i j-\frac{\sum_{i} \Sigma_{j} E(N P 3) i j}{m * n}\right)^{2}\right.}{m * n}}}+\frac{E(S w) i j-\frac{\sum_{i} \Sigma_{j} E(S w) i j}{m * n}}{\sqrt{\frac{\left(\sum_{i} \sum_{j}\left(E(S w) i j-\frac{\sum_{i} \sum_{j} E(S w) i j}{m * n}\right)^{2}\right.}{m * n}}}+\frac{E(H w) i j-\frac{\sum_{i} \Sigma_{j} E(H w) i j}{m * n}}{\sqrt{\frac{\left(\sum_{i} \sum_{j}\left(E(H w) i j-\frac{\sum_{i} \Sigma_{j} E(H w) i j}{m * n}\right)^{2}\right.}{m * n}}}+ \\
& \left.\frac{E(b i o) i j-\frac{\sum_{i} \sum_{j} E(b i o) i j}{m * n}}{\sqrt{\frac{\left(\sum_{i} \sum_{j}\left(E(b i o) i j-\frac{\sum_{i} \sum_{j} E(b i o) i j}{m * n}\right)^{2}\right.}{m * n}}}\right) * X i j
\end{aligned}
$$

- Objective function of Goal 2: The goal is the minimization of total improvement cost including environmental and social costs. Investment cost is the total cost for improvement of all processes and selected correspondence alternatives. Thus, the objective function of case 1 is given by Equation (19):

$$
G 2=\operatorname{Min}\left(\quad \sum_{i=1}^{n} \sum_{j=1}^{m i} C p i j * X i j+\sum_{i=1}^{n} \sum_{j=1}^{m i} C E i j * X i j+\sum_{i=1}^{n} \sum_{j=1}^{m i} C s i j * X i j+\mathrm{C}_{\mathrm{c}}\right)
$$

- $\quad$ : total number processes of the plant

- $\quad m_{i}$ : number of alternatives of process $P i, i=1 \ldots n$

- $j=1 \ldots m i$

- $\quad C_{i j}:$ investment cost of $X_{i j}$

- $\quad$ : : total cost for production line

- Objective function of Goal 3: The objective function for social dimension is: Maximizing positive social impacts minus negative social impacts. The social parameters are defined in table. The following different social impacts are quantified and combined together as one Objective function in Equation 15:

$$
\text { Equation } 15: G 3=\operatorname{Max} \quad \sum \sum\left(S_{i j}\right) x_{i j^{-}} \sum \sum\left(S_{i j}\right) x_{i j}
$$

By using equation (11) and equation (15), we have equation (16):

$$
\begin{aligned}
& G 3=\operatorname{Max} \sum \sum\left(\left(\frac{S(l r) i j-\frac{\sum_{i} \Sigma_{j} S(l r) i j}{m * n}}{\sqrt{\frac{\left(\sum_{i} \Sigma_{j}\left(S(l r) i j-\frac{\sum_{i} \Sigma_{j} S(l r) i j}{m * n}\right)^{2}\right.}{m * n}}}+\frac{S(d f) i j-\frac{\sum_{i} \Sigma_{j} S(d f) i j}{m * n}}{\sqrt{\frac{\left(\sum_{i} \Sigma_{j}\left(S(d f) i j-\frac{\sum_{i} \Sigma_{j} S(d f) i j}{m * n}\right)^{2}\right.}{m * n}}}+\frac{S(h r) i j-\frac{\sum_{i} \Sigma_{j} S(h r) i j}{m * n}}{\sqrt{\frac{\left(\sum_{i} \Sigma_{j}\left(S(h r) i j-\frac{\Sigma_{i} \Sigma_{j} S(h r) i j}{m * n}\right)^{2}\right.}{m * n}}}+\right.\right. \\
& \frac{S(t r) i j-\frac{\sum_{i} \Sigma_{j} S(t r) i j}{m * n}}{\sqrt{\frac{\left(\sum_{i} \sum_{j}\left(S(t r) i j-\frac{\sum_{i} \Sigma_{j} S(t r) i j}{m * n}\right)^{2}\right.}{m * n}}}+\frac{S(c s) i j-\frac{\sum_{i} \Sigma_{j} S(c s) i j}{m * n}}{\sqrt{\frac{\left(\sum_{i} \sum_{j}\left(S(c s) i j-\frac{\sum_{i} \sum_{j} S(c s) i j}{m * n}\right)^{2}\right.}{m * n}}}+\frac{S(a r) i j-\frac{\sum_{i} \sum_{j} S(a r) i j}{m * n}}{\sqrt{\frac{\left(\sum_{i} \sum_{j}\left(S(a r) i j-\frac{\sum_{i} \sum_{j} S(a r) i j}{m * n}\right)^{2}\right.}{m * n}}}+\frac{S(e l) i j-\frac{\sum_{i} \Sigma_{j} S(e l) i j}{m * n}}{\sqrt{\frac{\left(\sum_{i} \sum_{j}\left(S(e l) i j-\frac{\sum_{i} \sum_{j} S(e l) i j}{m * n}\right)^{2}\right.}{m * n}}}
\end{aligned}
$$




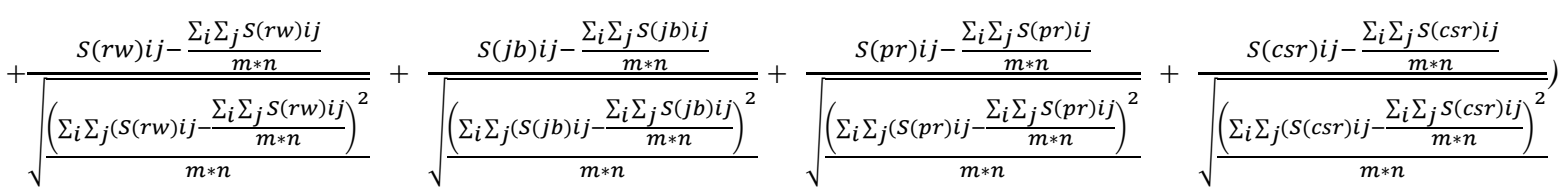

$$
\begin{aligned}
& -\left(\frac{S(a h) i j-\frac{\Sigma_{i} \Sigma_{j} S(a h) i j}{m * n}}{\sqrt{\frac{\left(\sum_{i} \sum_{j}\left(S(a h) i j-\frac{\sum_{i} \Sigma_{j} S(a h) i j}{m * n}\right)^{2}\right.}{m * n}}}++\frac{S(e h) i j-\frac{\sum_{i} \Sigma_{j} S(e h) i j}{m * n}}{\sqrt{\frac{\left(\sum_{i} \sum_{j}\left(S(e h) i j-\frac{\sum_{i} \sum_{j} S(e h) i j}{m * n}\right)^{2}\right.}{m * n}}}+\frac{S(c p) i j-\frac{\Sigma_{i} \Sigma_{j} S(c p) i j}{m * n}}{\sqrt{\frac{\left(\sum_{i} \Sigma_{j}\left(S(c p) i j-\frac{\sum_{i} \Sigma_{j} S(c p) i j}{m * n}\right)^{2}\right.}{m * n}}}+\right.
\end{aligned}
$$

$$
\left.\frac{S(p u) i j-\frac{\sum_{i} \Sigma_{j} S(p u) i j}{m * n}}{\left.\sqrt{\frac{\left(\sum_{i} \Sigma_{j}\left(S(p u) i j-\frac{\sum_{i} \Sigma_{j} S(p u) i j}{m * n}\right)^{2}\right.}{m * n}}\right)}\right) * X_{i j}
$$

\subsubsection{Constraints}

Always resources are limited and main constraints are related to limitation of resources. The main resources for every production lines are Budget and Time. Some other constraints are related to rules and regulation like as: Government limitation for Carbon Cap, Environmental Protection rules for using water and electricity, Social rules, Stockholders and management rules and so on.

\section{- Time Limitation:}

As the commitment of factory to customers for their Demand and also time limitation for production line, we define time constraint as in Equation (17).

$$
\sum_{i=1}^{n} \sum_{j=1}^{m i} T i j * X i j * D \leq 250
$$

- Tij: The time of doing process $\mathrm{Xij}$ in the scale of day;

- Total working Days for one year are 250 days.

- D: Total Demand for One year.

\section{- Budget Limitation:}

We consider the budget for decreasing environmental impact as $C_{0}$. Thus, total investment cost for environmental impact must be less than $\mathrm{C}_{\mathrm{o}}$. Constraint of investment cost can be formulated as Equations $(18,19,20)$ :

$$
C_{0} \geq\left(\sum_{i=1}^{n} \sum_{j=1}^{m i} C E i j * X i j+\mathrm{C}_{\mathrm{c}}\right)
$$

The budget of payment to Personal Staff for new job opportunities and training workers is $\mathrm{CS}_{\mathrm{o}}$, thus, the total costs of staff payment should be less than budget :

$$
C S_{0} \geq \sum_{i=1}^{n} \sum_{j=1}^{m i} C s i j * X i j * J B c i j
$$

In addition, total costs including investment for environmental impact and staff payment must be less than $\mathrm{S}_{\mathrm{o}}+$ $\mathrm{C}_{0}$. Constraint of investment cost can be formulated as Equation (17):

$$
\text { Equation 20: }\left(C S_{0}+C_{0}\right) \geq \sum_{i=1}^{n} \sum_{j=0}^{m i} C s i j * b i j * X i j+\sum_{i=1}^{n} \sum_{j=0}^{m i} C E i j * J B c i j * X i j
$$

We consider TB for total budget of production line and we have below constraint:

$$
\text { Equation 21: } T C \geq\left(\sum_{i=1}^{n} \sum_{j=1}^{m i} C p i j * X i j+\sum_{i=1}^{n} \sum_{j=1}^{m i} C E i j * X i j+\sum_{i=1}^{n} \sum_{j=1}^{m i} C s i j * X i j+\mathrm{C}_{\mathrm{c}}\right)
$$

- Environmental Limitation: 
If we consider the objective of environmental impact is ER and the existing situation is $\mathrm{E}_{0}$, Thus, total decreasing environmental impact is not less than ER. It means that $\mathrm{E}_{0} * \mathrm{X}_{\mathrm{ij}}-\mathrm{E}_{\mathrm{ij}} * \mathrm{X}_{\mathrm{ij}}$ should be less than ER. The constraint of environmental impact reduction potential can be formulated as Equation (22):

Equation 22: $\quad \mathrm{ER} \leq \quad\left(\left(\sum_{i}\left(\frac{E(\text { Energy }) i 0-\frac{\sum_{i} E(\text { Energy }) i 0}{n}}{\sqrt{\frac{\left(\sum_{i} E(\text { Energy }) i 0\right.}{\left.\frac{\sum_{i} E(\text { Energy }) i 0}{n}\right)^{2}}}}\right)+\sum_{i}\left(\frac{E(\text { Water }) i 0-\frac{\sum_{i} E(\text { Water }) i 0}{n}}{\sqrt{\left.\frac{\left(\sum_{i} E(\text { Water }) i 0-\frac{\Sigma_{i} E(\text { Water }) i 0}{n}\right.}{n}\right)^{2}}}\right)+\right.\right.$ $\sum_{i}\left(\frac{E(\mathrm{CO} 2) i 0-\frac{\sum_{i} E(\mathrm{CO} 2) i 0}{n}}{\sqrt{\frac{\left(\sum_{i} E(\mathrm{CO} 2) i 0-\frac{\sum_{i} E(\mathrm{CO}) i 0}{n}\right)^{2}}{n}}}\right)+\sum_{i}\left(\frac{E(\mathrm{CH} 4) i 0-\frac{\sum_{i} E(\mathrm{CH} 4) i 0}{n}}{\sqrt{\frac{\left(\sum_{i} E(\mathrm{CH} 4) i 0-\frac{\sum_{i} E(\mathrm{CH} 4) i 0}{n}\right)^{2}}{n}}}\right)+\sum_{i}\left(\frac{E(\mathrm{~N} 2 \mathrm{O}) i 0-\frac{\sum_{i} E(\mathrm{~N} 2 \mathrm{O}) i 0}{n}}{\sqrt{\frac{\left(\sum_{i} E(\mathrm{~N} 2 O) i 0-\frac{\sum_{i} E(\mathrm{~N} 2 \mathrm{O}) i 0}{n}\right)^{2}}{n}}}\right)$

$+\sum_{i}\left(\frac{E(H F C S) i 0-\frac{\sum_{i} E(H F C S) i 0}{n}}{\sqrt{\frac{\left(\sum_{i} E(H F C S) i 0-\frac{\Sigma_{i} E(H F C S) i 0}{n}\right)^{2}}{n}}}\right)+\sum_{i}\left(\frac{E(P F C S) i 0-\frac{\sum_{i} E(P F C S) i 0}{n}}{\sqrt{\frac{\left(\sum_{i} E(P F C S) i 0-\frac{\Sigma_{i} E(P F C S) i 0}{n}\right)^{2}}{n}}}\right)+\sum_{i}\left(\frac{E(N P 3) i 0-\frac{\sum_{i} E(N P 3) i 0}{n}}{\sqrt{\frac{\left(\sum_{i} E(N P 3) i 0-\frac{\Sigma_{i} E(N P 3) i 0}{n}\right)^{2}}{n}}}\right)+$ $\sum_{i}\left(\frac{E(S w) i 0-\frac{\sum_{i} E(S w) i 0}{n}}{\sqrt{\frac{\left(\sum_{i} E(S w) i 0-\frac{\sum_{i} E(S w) i 0}{n}\right)^{2}}{m * n}}}\right)+\sum_{i}\left(\frac{E(H w) i 0-\frac{\sum_{i} E(H w) i 0}{n}}{\sqrt{\frac{\left(\sum_{i} E(H w) i 0-\frac{\sum_{i} E(H w) i 0}{n}\right)^{2}}{n}}}\right)+\sum_{i}\left(\frac{E(b i o) i 0-\frac{\sum_{i} E(b i o) i 0}{n}}{\sqrt{\frac{\left(\sum_{i} E(b i o) i 0-\frac{\sum_{i} E(b i o) i 0}{n}\right)^{2}}{n}}}\right)$ ( $\sum_{i} \sum_{j}\left(\frac{E(\text { Energy }) i j-\frac{\sum_{i} \Sigma_{j} E(\text { Energy }) i j}{m * n}}{\sqrt{\frac{\left(\sum_{i} \Sigma_{j}\left(E(E n e r g y) i j-\frac{\Sigma_{i} \Sigma_{j} E(E n e r g y) i j}{m * n}\right)^{2}\right.}{m * n}}}+\frac{E(\text { Water }) i j-\frac{\sum_{i} \Sigma_{j} E(\text { water }) i j}{m * n}}{\sqrt{\frac{\left(\sum_{i} \Sigma_{j}\left(E(\text { water }) i j-\frac{\Sigma_{i} \Sigma_{j} E(\text { water }) i j}{m * n}\right)^{2}\right.}{m * n}}}+\frac{E(C O 2) i j-\frac{\sum_{i} \Sigma_{j} E(C O 2) i j}{m * n}}{\sqrt{\frac{\left(\sum_{i} \Sigma_{j}\left(E(C O 2) i j-\frac{\Sigma_{i} \Sigma_{j} E(C O 2) i j}{m * n}\right)^{2}\right.}{m * n}}}+\right.$ $\frac{E(N 2 O) i j-\frac{\sum_{i} \Sigma_{j} E(N 2 O) i j}{m * n}}{\sqrt{\frac{\left(\sum_{i} \Sigma_{j}\left(E(N 2 O) i j-\frac{\Sigma_{i} \Sigma_{j} E(N 2 O) i j}{m * n}\right)^{2}\right.}{m * n}}}+\frac{E(H F C S) i j-\frac{\sum_{i} \sum_{j} E(H F C S) i j}{m * n}}{\sqrt{\frac{\left(\sum_{i} \Sigma_{j}\left(E(H F C S) i j-\frac{\sum_{i} \Sigma_{j} E(H F C S) i j}{m * n}\right)^{2}\right.}{m * n}}}+\frac{E(P F C S) i j-\frac{\sum_{i} \Sigma_{j} E(P F C S) i j}{m * n}}{\sqrt{\frac{\left(\sum_{i} \Sigma_{j}\left(E(P F C S) i j-\frac{\sum_{i} \sum_{j} E(P F C S) i j}{m * n}\right)^{2}\right.}{m * n}}}+$ $\frac{E(N P 3) i j-\frac{\sum_{i} \Sigma_{j} E(N P 3) i j}{m * n}}{\sqrt{\frac{\left(\sum_{i} \Sigma_{j}\left(E(N P 3) i j-\frac{\Sigma_{i} \sum_{j} E(N P 3) i j}{m * n}\right)^{2}\right.}{m * n}}}+\frac{E(S w) i j-\frac{\sum_{i} \Sigma_{j} E(S w) i j}{m * n}}{\sqrt{\frac{\sum_{i} \Sigma_{j}\left(E(S w) i j-\frac{\Sigma_{i} \sum_{j} E(S w) i j}{m * n}\right)^{2}}{m * n}}}+\frac{E(H w) i j-\frac{\sum_{i} \Sigma_{j} E(H w) i j}{m * n}}{\sqrt{\frac{\sum_{i} \Sigma_{j}\left(E(H w) i j-\frac{\Sigma_{i} \Sigma_{j} E(H w) i j}{m * n}\right)^{2}}{m * n}}}+$ $\left.\left.\frac{E(b i o) i j-\frac{\sum_{i} \sum_{j} E(b i o) i j}{m * n}}{\sqrt{\frac{\left(\sum_{i} \Sigma_{j}\left(E(b i o) i j-\frac{\sum_{i} \Sigma_{j} E(b i o) i j}{m * n}\right)^{2}\right.}{m * n}}}\right) * X i j\right)$

\section{Social Limitation:}

(Stindt, 2017) defined that the constraints in social dimension are related to human right, labor practice and decent work and Social Life Cycle Assessment (SLCA) of Products. The human rights in different countries are defined in the labor rules and conditions. For decent work, normally is 160 hours/month and 250 days in the year. We formulate constraint as per below for working time, we consider NYW parameter as normal yearly work (Man/Day for one year):

$$
\text { Equation 23: } \sum_{i=1}^{n} \sum_{j=1}^{m i}(S j b * X i j * T i j) \leq N Y W=250
$$




\section{- Definition of Decision Variables limitations:}

$\mathrm{x}_{\mathrm{ij}}$ is the binary variable, $\mathrm{x}_{\mathrm{ij}}=0,1$, if $\mathrm{X}_{\mathrm{ij}}$ The following constraints are related to the $\mathrm{X}_{\mathrm{ij}}$ which are decision variables in the model.

$$
\begin{gathered}
\text { Equation 24: } \sum_{j=0}^{m i} X i j=1 \\
\text { Equation 25: Xij= }\{0,1\} \\
\text { Equation 26: : } \sum_{i=1}^{n} \sum_{j=1}^{m i} X i j=\mathrm{n}
\end{gathered}
$$

The model for Sustainable Production Line is developed now. According to this way, we can develop new mathematical model for other modules of SSCM.

\section{Conclusion}

The research of this paper is in the context of designing production processes appropriate to environmental and social impacts. The question of this research is how we can design sustainable production line which has the minimum bad effects on environmental and social and fulfill the minimum economic requirements. For this design, the literature on environmental and social impacts has been reviewed at first, and the closest research framework to this topic has been SSCM. By studying the structures and models common in SSCM and modeling, we came up with the Sustainable Production Line model for designing production line processes. This model uses the structure introduced in the SSCM and based on the minimum environmental impact and the maximum social benefits.

Using the literature review, environmental and social impact parameters are identified in tables. Normal distribution is used to apply the parameters in the mathematical model and to match them. Based on the mathematical models of integer programming and multi goal programming the basic mathematical model of SPL was developed. The variables of decision making are the alternatives of processes for production lines which are impacts on costs of production lines, also have social and environmental impacts.

It is recommended to develop this model in different modules of supply chain management like Transportation and Logistics processes, Purchasing and procurement, Design, and so on. And fit the relevant environmental and social parameters into the model for each module. In light of the conclusions above, the study recommends that making mathematical model for the whole SSCM process with considering Environmental and social parameters.

\section{Acknowledgment}

This research is funded by National Natural Science Foundation of China under Grand Number: 71832001. Also, thanks Department of Logistics and Electronic Commerce, Glorious Sun School of Business and Management, Shanghai Donghua University for supporting us in this project.

\section{References}

Agan, Y., Cemil, K. C., Acar, M., \& Atif, A. (2016). The relationships between Corporate cosial responsibility, environmental supplier, and firm performance. Journal of Cleaner Production, 112, 1872-1881. https://doi.org/10.1016/j.jclepro.2014.08.090

Aktin, T., \& Gergin, Z. (2016). Mathematical modeling of sustainable procurement strategies: three case studies. Journal of Cleaner Production, 113, 767-780. https://doi.org/10.1016/j.jclepro.2015.11.057

Alinso, L., Rubino, E. M., Agustina, B., \& Domingo, R. (2017). Latest clean manufacturing trends applied to a world class manufacturing management for improving logistics and environmental performance. Procedia Manufacturing , 13, 1151-1158. https://doi.org/10.1016/j.promfg.2017.09.177

Allaoui, H., Guo, Y., Choudhary, A., \& Bloemho-Rawaard, J. M. (2017369-384). Sustainable agro-food supply chain design using two-stage hybrid multi-objective decision-making approach. Computer and Operation Research, 89. https://doi.org/10.1016/j.cor.2016.10.012

Arampantzi, C., \& Minis, I. (2017). A new model for designning sustainable supply chain networks and its application at a global manufacturer. Journal of Cleaner Production, 276-292. https://doi.org/10.1016/j.jclepro.2017.03.164

Awasthi, A., Govindan, K., \& Gold, S. (2018). Multi-tier sustainable global supplier selection using a fuzzy 
AHP-VIKOR based approach. International Journal of Production Economics, 195, 106-117. https://doi.org/10.1016/j.ijpe.2017.10.013

Azadi, M., Jafarian, M., Farzinpoor, S. R., \& Mirhedayatian, S. M. (2015). A new fuzzy DEA model for evaluation of efficiency and effectiveness of suppliers in sustainable supply chain management contex. Computer \& Operation Research, 274-285. https://doi.org/10.1016/j.cor.2014.03.002

Azimifard, A., Moosavirad, S. H., \& Ariafar, S. (2018). Selection sustainable supplier countries for Iran's steel industry at three levels by using AHP nad TOPSIS methods. Resource Policy, 57, 30-44. https://doi.org/10.1016/j.resourpol.2018.01.002

Badiezadeh, T., Farzipoor, S. R., \& Samavati, T. (2017). Assessing sustainability of supply chain by double frontier network DEA: A big data approach. Computer and Operation Research, 1-7.

Bai, L., Xie, M., Zhang, Y., \& Qiao, Q. (2017). Pollution prevention and control measures for the bottom blowing furnace of a lead-smelting process, based on a mathematical model and simulation. Journal of Cleaner Production, 159, 432-445. https://doi.org/10.1016/j.jclepro.2017.05.057

Boudaghi, E., \& Farzipoor S. R. (2018). Developing a novel model of data envelopment analysis-discriminant analysis of predicting group membership of suppliers in sustainable supply chain. Computer and Operation Research, 89, 348-359. https://doi.org/10.1016/j.cor.2017.01.006

Costanza, R., \& Patten, B. (1995). Commentary definning and predicting sustainability. Ecological Economics. 15, 193-196. https://doi.org/10.1016/0921-8009(95)00048-8

Devika, K., Jafarian, A., \& Nourbakhsh, V. (2014). Designing a sustainable closed-loop supply chain network based on triple bottom line approach: A comparision of metaheuristics hybridization techniques. European Journal of Operation Research, 235, 495-615. https://doi.org/10.1016/j.ejor.2013.12.032

Govindan, K., Shankar, M., \& Devika, K. (2018). Supplier selection based on corporate social responsibility practices. International Journal of Production Economics, 353-379. https://doi.org/10.1016/j.jpe.2016.09.003

Gred, J. H., \& Brandenburg, M. (2018). A sustainable aggregate production planning model for the chemical process industry. Computers and Operation Research, 94, 154-168. https://doi.org/10.1016/j.cor.2017.12.011

Hatami-Marbini, A., Agrell, J., Tavana, M., \& Khoshnevis, P. (2017). A flexible cross-efficiency fuzzy data envelopment analysis model for sustainable sourcing. Journal of Cleaner Production, 142, 2761-2779. https://doi.org/10.1016/j.jclepro.2016.10.192

Hsueh, C. (2015). A bilevel programming model for Corporate social responsibility. Transportation Research, Part E73. https://doi.org/10.1016/j.tre.2014.11.006

Izadikhah, M., Farzipoor, S. R., \& Ahmadi, K. (2017). How to assess sustainability of suppliers in volume discount contex? A new data envelopment analysis approach. Transportation Research, Part D, 102-121. https://doi.org/10.1016/j.trd.2016.11.030

Ji, X., Wu, J., \& Zhu, Q. (2016). Eco-design of transportation in sustainable supply chain management, A DEA like method. Transportatio Research, Part D, 451-459. https://doi.org/10.1016/j.trd.2015.08.007

Jones, D. F., \& Tamiz, M. (2002). Chapter 3-Goal Programming in the period 1990-2000. In M. G. Ehrgott, Multiple Criteria Optimization, State of the Art Annotated Bibliographic Survey (Vol. 52). Kluwer Academic Publishers, USA: International Series in Operations Research \& Management science.

Lee, T., \& Nam, H. (2016). An Empirical study on the impact of Individual and organizational supply chain oriented on supply chain management. Journal of shipping and Logistics, 249-255. https://doi.org/10.1016/j.ajs1.2016.12.009

Luthra, S., Govindan, K., Kannan, D., Mangla, S. K., \& Garg, C. P. (2017). An integrated framework for sustainable supplier selection and evaluation in supply chain. Journal of Cleaner Production, 140, 1686-1698. https://doi.org/10.3390/su10072543

Mathivathanan, D., Govindan, K., \& Noorul Haq, A. (2017). Exploring the impact of dynamic capabilities on sustainable supply. Journal of Cleaner Production, 147, 637-653.

https://doi.org/10.1016/j.jclepro.2016.07.119

Motevali, H. S., Torabi, S. A., \& Ghasemi, R. (2016). An integrated approach for performance evaluation in 
sustainable supply chain network (with a case study). Journal of Cleaner Production, 579-597. https://doi.org/10.1016/j.jclepro.2016.07.119

Popovic, T., Barbosa-Poyoa, A., Kraslawski, A., \& Carvalho, A. (2018). Quantitative indicators for social sustainability assessment of supply. Journal of Cleaner Production, 137, 748-768. https://doi.org/10.1016/j.jclepro.2018.01.142

Raj, A., Biswas, I., \& Srivastava, S. K. (2018). Designing supply contracts for the sustainable supply chain using game theory. Journal of Cleaner Production, 185, 275-284. https://doi.org/10.1016/j.jclepro.2018.03.046

Sazvar, Z., Rahmani, M., \& Govindan, K. (2018). A sustainable supply chain for organic, conventional agro-food products: The role of demand substitution, climate change and public Health. Jounal of Business Strategy and Environment, 194, 564-583. https://doi.org/10.1016/j.jclepro.2018.04.118

Seuring, S., \& Muller, M. (2008). Core issues in sustainable supply chain management- a Delphi study. Bus. Strategy Environ., 17(8), 455-466. https://doi.org/10.1002/bse.607

Song, M., Cen, L., Zheng, Z., Fisher, R., Liang, X., Wang,Y., \& Huisingh, D. (2017). How would big data support societal development and environmental sustainability? Journal of Cleaner Production, 142, 489-500. https://doi.org/10.1016/j.jclepro.2016.10.091

Stindt, D. (2017). A generic planning approach for sustainable supply chain management - How to integrate concepts and methods to address the issues of sustainability? Journal of Cleaner Production, 153, 146-163. https://doi.org/10.1016/j.jclepro.2017.03.126

Tajbakhsh, A., \& Hassini, E. (2015). A data envelopment analysis approach to evaluate sustainability in supply chain networks. Journal of Cleaner Production, 142, 74-85. https://doi.org/10.1016/j.jclepro.2014.07.054

Tran, T. V., Schnitzer, H., Braunegg, G., \& Lee, H. T. (2017). Development of an optimization mathematical model by applying an integrated environmental indicator for selecting alternatives in cleaner production program. Journal of Cleaner Production, 154, 295-308. https://doi.org/10.1016/j.jclepro.2017.04.009

Tsalis, T. A., \& Evangelinos, K. L. (2019). A framework to measure corporate sustainability performance: A strong sustainability-based view of firm. Sustainable Production and Consumption, 18, 1-18. https://doi.org/10.1016/j.spc.2018.10.004

Tseng, S. C., \& Huang, S. W. (2014). A stratgic decision-making model considering the social costs of carbon dioxide emission for sustainable supply chain management. Journal of Environmental Management, 133, 315-322. https://doi.org/10.1016/j.jenvman.2013.11.023

Yousefi, S., Soltani, R., Farzipoor, S. R., \& Pishvaee, M. S. (2017). A robust fuzzy possibilistic programming for a new network GP-DEA model to evaluate sustainable supply chain. Journal of Cleaner Production, 166, 537-549. https://doi.org/10.1016/j.jclepro.2017.08.054

Zhou, G., \& Zhang, Y. (2017). Integration and Consolidation in air freight shipment planning: An economic and environmental perspective. Journal of Cleaner Production, 166, 1381-1394. https://doi.org/10.1016/j.jclepro.2017.07.145

Zhu, W., \& He, Y. (2017). Green product design in supply chain under competition. European Journal of Operational Research, 258, 165-180. https://doi.org/10.1016/j.ejor.2016.08.053

\section{Copyrights}

Copyright for this article is retained by the author(s), with first publication rights granted to the journal.

This is an open-access article distributed under the terms and conditions of the Creative Commons Attribution license (http://creativecommons.org/licenses/by/4.0/). 